\title{
Comparators for Voltage Transformer Calibrations at NBS
}

\author{
Wilbur C. Sze
}

\author{
(July 28, 1965)
}

\begin{abstract}
An inductive comparator is described for measuring ratio-correction-factor and phase-angle error of voltage transformers by comparison with reference transformers. The simple circuit utilizes a resistance-capacitance network and an inductive voltage divider. The relative magnitude of the ratiocorrection-factors is obtained from the dial readings of the inductive voltage divider, and the phase angle between the voltage phasors is equal to $\pm \omega \mathrm{CR}$. The accuracy of these measurements at 60 and 400 hertz is within 2 parts-per-million for ratio and within 10 microradians for phase angle at the limits of the phase-angle range of \pm 38 milliradians. Only a few ranges of reference transformers are required since fractional ratios from $1 / 1$ to $1 / 4$ may be measured as readily as ratios near unity, without diminution in accuracy. A general discussion of a resistive comparator, which has been in continuous operation at NBS since 1939, is included.
\end{abstract}

Key Words: Voltage divider, inductive voltage divider, resistive voltage divider, voltage transformer calibration, ratio-correction-factor, phase-angle errors, voltage transformer comparator, voltage-transformer-test-set, comparator, test-set, calibration.

\section{Introduction}

The method most widely used by public utilities and standardizing laboratories to determine the ratio and phase angle of voltage transformers is by comparison with calibrated reference transformers. However, it is a relative rather than an absolute method, in that the accuracy of the results obtained depends upon the knowledge of accuracy of the reference transformers [1]. ${ }^{1}$

At the NBS laboratories, a group of reference transformers is available by means of which test transformers having any ratio from 1:1 to 2000:1 can be calibrated. The accurate initial establishment of the voltage ratio and phase angle for these reference transformers has been discussed elsewhere and will not be repeated here $[2,3]$.

The purposes of this paper are (1) to describe the resistive comparator which has been in continuous operation since 1939; (2) to describe a new inductive comparator; and (3) to analyze various factors affecting the accuracy with which measurements can be made.

\section{Resistive Comparator}

This comparator was designed and constructed at NBS by F. K. Harris and has been in continuous operation since 1939, except for a very brief period during which new switches were installed to replace the worn out original ones $[4,5]$.

The schematic circuit of this comparator is shown in figure $1 .^{2}$ The in-phase voltage balance condition is achieved by adjusting the tap of the resistive divider, and the quadrature voltage, by varying the mutual inductor, $M$.

\footnotetext{
Figures in brackets indicate the literature references at the end of this paper.

${ }^{2}$ Simplified by not showing the shielding arrangements around each segment of $R_{s}$.
}

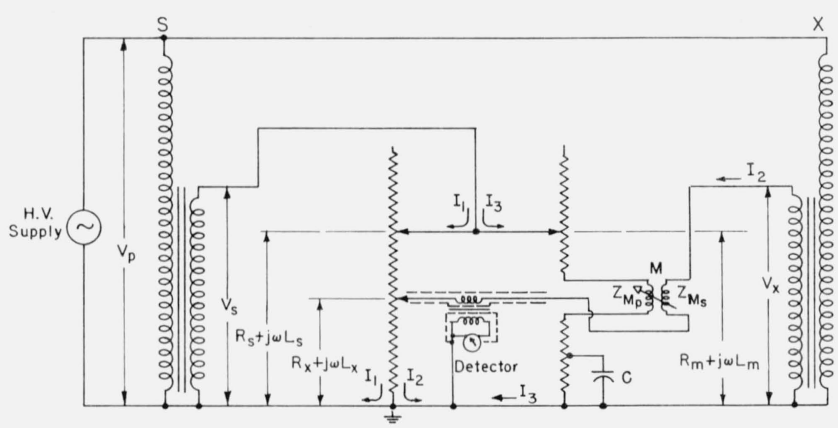

FigURE 1.

\subsection{Description of Components}

The $R_{s}$ and $R_{m}$ circuits are supplied from the secondary of transformer $S$. Each can be varied from 2000 to $4000 \Omega$ in increments of $100 \Omega$. The exact values are determined by the ratios of the test and reference transformers. The $100-\Omega$ and $1000-\Omega$ unit resistors of $R_{s}$ are of woven wire and mounted on ceramic forms. Thus the residual, $L_{s}$, of $R_{s}$ is reduced to a minimum [6]. The $R_{m}$ circuit, nominally equal to $R_{s}$, include the primary circuit of $M$ which has a d-c resistance of $200 \Omega$. The capacitor, $C$, shunting the lower portion of the resistance, serves to compensate for the self-inductance introduced by $M$ [5]. Multiple taps are brought out from $R_{m}$ to supply the shield potential of corresponding sections of $R_{s}$ and of the detector circuit [7]. The shielding is shown in figure 4.

As shown in figure 2, the portion of the $R_{s}$ circuit between 1800 and $2000 \Omega$ carries a continuously ad. justable tap, $R_{x}$, which is connected through the detector circuit and $M$ to the secondary of the test transformer. 


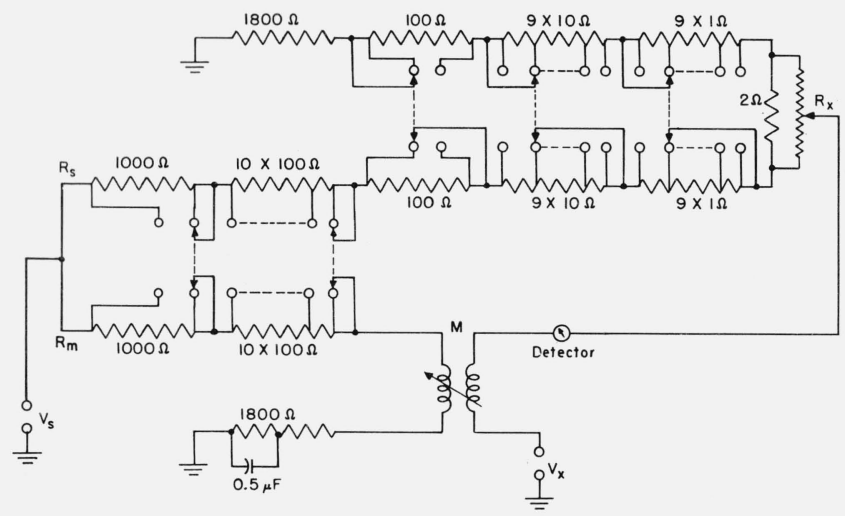

Figure 2.

The mutual inductor used in this circuit consists of a $10 \times 20 \mathrm{mH}$ per section unit $( \pm 200 \mathrm{mH}$ maximum) and a continuously adjustable inductometer $( \pm 25$ $\mathrm{mH}$ maximum) $[8,9]$.

Both the $R_{s} / R_{x}$ ratio of the resistive divider and the mutual inductance, $M$, are periodically redetermined to insure that maximum accuracy is maintained for transformer testing. The residual reactances, $L_{s}$ and $L_{x}$, were also accurately determined at the time the comparator was built.

The design is such that the resistors must dissipate considerable power. Therefore, the entire resistive divider, $R_{s}$ and $R_{m}$, is immersed in oil to minimize the effect of temperature coefficient on the $R_{s} / R_{x}$ ratio.

The detector circuit consists of a doubly shielded impedance-matching transformer (1000-4000 turns) and a wave-analyzer used as a tuned null detector.

\subsection{Comparator Desk}

Figure 3 shows the general layout of the desk. The resistive divider is located to the right with the $R_{s}$ adjustment dials in the back and the $R_{x}$ dials in front. The step mutual inductor and the inductometer are located in the left portion of the desk. The detector is in the rear right. Since this comparator is adaptable for calibrating voltage-transformer-test-sets, the panel shown at the lower left side of the desk is for arranging the connections for calibrating either transformers or test-sets. Figure 4 shows the internal wiring of the resistive divider and the individual shields around units of $R_{s}$.

\subsection{Theoretical Relations}

The equations for obtaining the ratio-correctionfactor and phase-angle error are developed as follows. By definition, the voltage ratio of a transformer is the ratio of the primary terminal voltage to the secondary

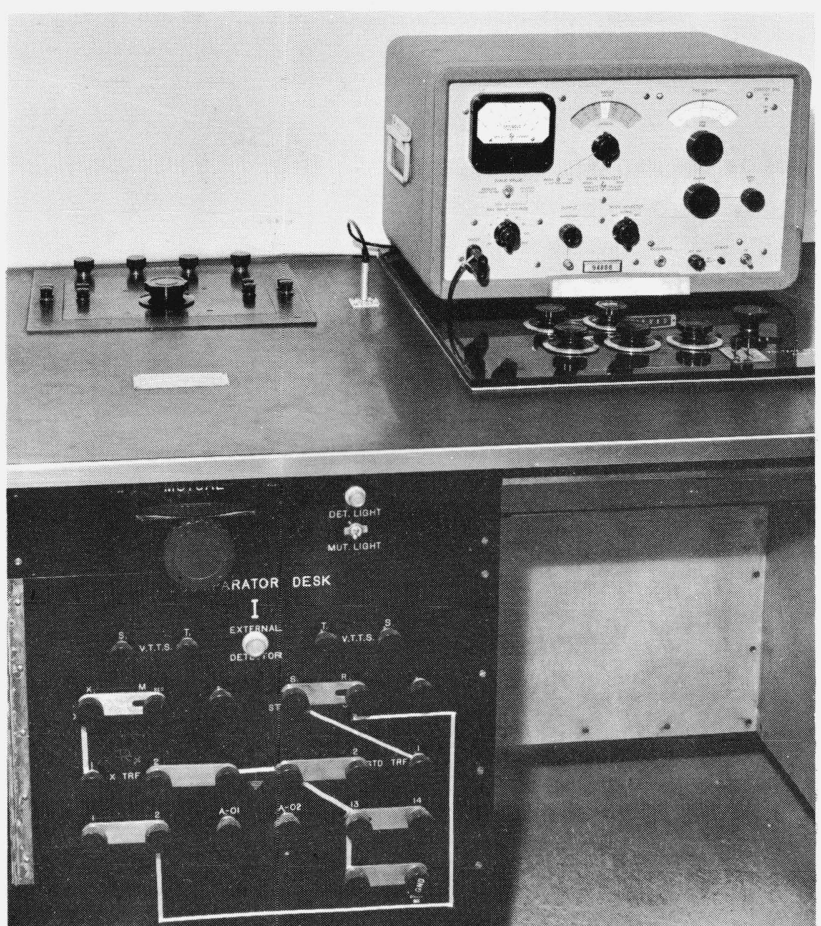

Figure 3.

terminal voltage so that

$$
\frac{\left|V_{\text {Pri }}\right|}{\left|V_{\text {Sec }}\right|}=N^{\prime}=N f
$$

where $N^{\prime}$ is the actual voltage ratio, $N$ the nominal ratio, and $f$ the ratio-correction-factor. If $\theta$ represents the angle between the primary and reversed secondary voltage phasors, then

$$
V_{s}=\frac{\left|V_{p}\right|}{N_{s}^{\prime}}\left(\cos \theta_{s}+j \sin \theta_{s}\right)
$$

and $^{3}$

$$
V_{x}=\frac{\left|V_{p}\right|}{N_{x}^{\prime}}\left(\cos \theta_{x}+j \sin \theta_{x}\right)
$$

By inspection of figure 1 , the following mesh current equations are obtained

$$
\begin{aligned}
& V_{s}=I_{1} Z_{s}+I_{2} Z_{x} \\
& V_{x}=I_{1} Z_{x}+I_{2}\left(Z_{x}+Z_{M s}\right)+I_{3} j \omega M \\
& V_{s}=I_{2} j \omega M+I_{3} Z_{m}
\end{aligned}
$$

${ }^{3}$ Subscripts $s$ and $x$ denote that the symbols refer to the reference or test transformers, respectively. 


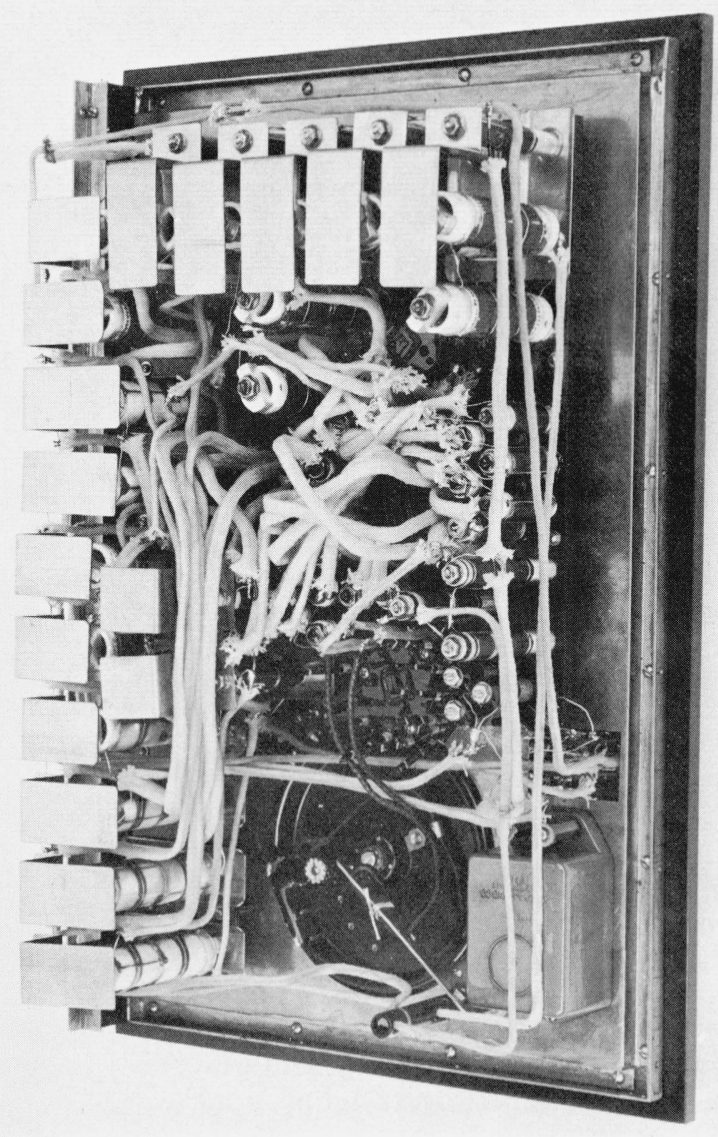

FigURE 4.

$I_{2}=0$ when a null is indicated on the detector, then

$$
V_{s}=I_{1} Z_{s}=I_{3} Z_{m}
$$

and

$$
V_{x}=V_{s}\left(\frac{Z_{x}}{Z_{s}}+\frac{j \omega M}{Z_{m}}\right)
$$

Since $Z_{m}=R_{m}$ by the addition of $C$, and $R_{m}=R_{s}$ nominally, the balance equation becomes

$$
\begin{aligned}
& \frac{\left(\cos \theta_{x}+j \sin \theta_{x}\right)}{N_{x} f_{x}}=\frac{\left(\cos \theta_{s}+j \sin \theta_{s}\right)}{N_{s} f_{s}} \\
&\left\{\frac{R_{x}}{R_{s}}\left[1+j \omega\left(\frac{L_{x}}{R_{x}}-\frac{L_{s}}{R_{s}}+\frac{M}{R_{x}}\right)\right]\right\}
\end{aligned}
$$

and on separation

$$
\begin{array}{r}
f_{x}=\frac{N_{s} R_{s}}{N_{x} R_{x}}\left(\frac{f_{s} \cos \theta_{x}}{\cos \theta_{s}-\sin \theta_{s} \omega\left(\frac{L_{x}}{R_{x}}-\frac{L_{s}}{R_{s}}+\frac{M}{R_{x}}\right)}\right) \\
\approx \frac{N_{s} R_{s}}{N_{x} R_{x}}+\left(f_{s}-1\right)+\frac{1}{2}\left(\theta_{s}^{2}-\theta_{x}^{2}\right)
\end{array}
$$

and ${ }^{4}$

$$
\theta_{x}=\theta_{s}+\omega\left(\frac{L_{x}}{R_{x}}-\frac{L_{s}}{R_{s}}+\frac{M}{R_{x}}\right) \text { radians }
$$

As previously indicated, $R_{s}$ and $R_{m}$ circuits constitute a burden on the reference transformer. Therefore, $f_{s}$ and $\theta_{s}$ are determined with these burdens.

The design of the divider is such that the balance conditions require

$$
\frac{N_{x}^{\prime}}{N_{s}^{\prime}}=\frac{R_{s}}{R_{x}} \geqslant 1,
$$

this requirement is met by using reference transformers whose voltage ratios have been adjusted to values 2 percent less than the integral values usually encountered as nominal ratios in instrument transformers.

The minimum resolution of this setup is such that the precision for ratio measurements is $2 \mathrm{ppm}$ and for phase angle, $15 \mu \mathrm{rad}$ at 25 and $60 \mathrm{~Hz}$. However, susceptibility of the mutual inductor to the gradients of stray magnetic fields, large residuals and uncertainty in ratio calibration of the resistive divider limit the accuracy of these measurements to $10 \mathrm{ppm}$ and 60 $\mu \mathrm{rad}$, respectively. These errors increase rapidly with frequency. Therefore, this comparator is not suitable for accurate calibrations beyond $60 \mathrm{~Hz}$.

\section{Inductive Comparator}

This comparator avoids a number of the objectionable features of the resistive comparator. The shielding is simple and is easily accomplished; the method is capable of extension to cover the audio-frequency range; the apparatus is less bulky and requires very little power for operation; the components are inherently more accurate and stable and readily available commercially; the ratio difference is indicated directly on the decade dials; and the set (except the detector) is self-contained in one portable case.

The schematic diagram of the circuit is shown in figure 5 , which indicates the positions of resistor, $R$, and detector when the voltage phasor of the test transformer, $X$, leads that of the reference transformer, $S$. For lagging angles, the positions of $R$ and detector are

\footnotetext{
${ }^{4}$ The $\left(f_{s}-1\right)$ and $\theta_{s}$ for the NBS reference transformers are very small. Therefore, the approximations made are justifiable.
}

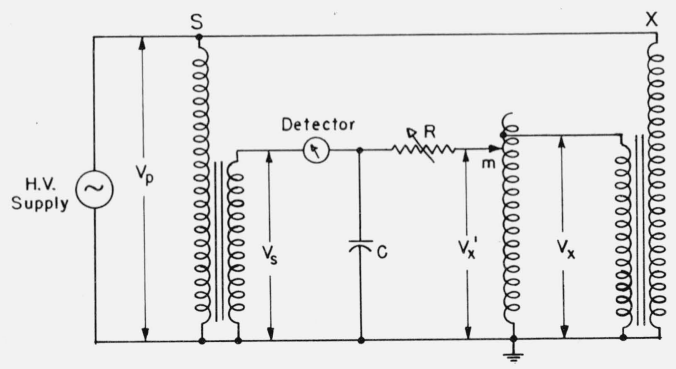

FIGURE 5. 
simply interchanged. The in-phase voltage balance is obtained by adjusting the tap on the inductive voltage divider, and the quadrature voltage balance is obtained by adjusting either the resistor or the capacitor.

\subsection{Theoretical Relations}

As previously defined, the voltage ratio is

$$
\begin{gathered}
\frac{\left|V_{\text {Pri }}\right|}{\left|V_{\text {Sec }}\right|}=N^{\prime}=N f, \\
V_{s}=\frac{\left|V_{p}\right|}{N_{s} f_{s}}\left(\cos \theta_{s}+j \sin \theta_{s}\right),
\end{gathered}
$$

and

$$
V_{x}=\frac{\left|V_{p}\right|}{N_{x} f_{x}}\left(\cos \theta_{x}+j \sin \theta_{x}\right)=\frac{V_{x}^{\prime}}{m},
$$

$m$ being the actual ratio of the inductive voltage divider. Corrections to the ratios and the phase angles of the inductive voltage divider used are insignificant and can be neglected. Then from figure 5 ,

$$
\frac{V_{s}}{V_{x}^{\prime}}=\frac{1-j \omega C R}{1+\omega^{2} C^{2} R^{2}} \approx 1-\omega^{2} C^{2} R^{2}-j \omega C R
$$

The balance equation of the circuit becomes

$$
\frac{N_{x} f_{x}\left(\cos \theta_{s}+j \sin \theta_{s}\right)}{m N_{s} f_{s}\left(\cos \theta_{x}+j \sin \theta_{x}\right)}=1-\omega^{2} C^{2} R^{2}-j \omega C R
$$

and on separation and by neglecting second order terms

$$
\theta_{x}=\frac{N_{x} f_{x}}{m N_{s} f_{s}}\left(\theta_{s}+\omega C R+\theta_{s} \omega^{2} C^{2} R^{2}\right) \approx \theta_{s}+\omega C R
$$

radians, and

$$
\begin{array}{r}
f_{x}=\frac{m N_{s} f_{s}}{N_{x}}\left(\frac{\cos \theta_{x}-\omega^{2} C^{2} R^{2}+\omega C R \sin \theta_{x}}{\cos \theta_{s}}\right) \\
\approx \frac{m N_{s}}{N_{x}}+\left(f_{s}-1\right)-\frac{1}{2}(\omega C R)^{2}
\end{array}
$$

For relative lagging phase angle, with the detector and resistor, $R$, interchanged,

$$
\begin{aligned}
\theta_{x} & \approx \theta_{s}-\omega C R \text { radians, and } \\
f_{x} & \approx \frac{m N_{s}}{N_{x}}+\left(f_{s}-1\right)+\frac{1}{2}(\omega C R)^{2}
\end{aligned}
$$

\subsection{Loading Effects}

Since the input impedance of the inductive voltage divider at $60 \mathrm{~Hz}$ is in excess of $0.8 \mathrm{M} \Omega$ (nearly 1.00 power factor), the effect of loading of the $f_{x}$ and $\theta_{x}$ of the test transformer can be expressed as

$$
\begin{aligned}
& \frac{\left(\begin{array}{c}
\text { Equivalent Total Impedance Referred } \\
\text { to Transformer Secondary }
\end{array}\right)}{(\text { External Impedance })} \\
& =\left(\text { Correction to } f_{x} \text { and } \theta_{x}\right) .
\end{aligned}
$$

A transformer with an equivalent resistance of $1 \Omega$ (which is easily measurable) will result in a correction of approximately $1 \mathrm{ppm}$ [5].

The effect of loading by the quadrature balancing network on either the inductive voltage divider or the reference transformer is usually insignificant. At the limits of phase-angle range of $\pm 37.8 \mathrm{mrad}$ (130 min), the correction to ratio and phase angle of the inductive voltage divider is $\sim 1 \mathrm{ppm}$ and $<4 \mu \mathrm{rad}$, respectively; or, to the reference transformer, $<<1$ ppm and $<<1 \mu$ rad.

\subsection{Description of Components}

All the components in this circuit are readily available commercially or accessible in most testing laboratories. In figure $5, R$ is a $0-10 \mathrm{k} \Omega, 10$-turn, continuously adjustable resistor with small residual reactance. $C$ is a $10 \times 1 \mathrm{nF}$ decade capacitor with a dissipation factor of $<0.0003$. Both $R$ and $C$ were calibrated before and after assembly. The deviations from nominal values were found to be within $5 \Omega$ and $5 \mathrm{pF}$, respectively.

The inductive voltage divider is a six-decade unit designed for optimum performance at $60 \mathrm{~Hz}^{5}$ For tests at higher frequencies (up to $1 \mathrm{kHz}$ ), it can easily be replaced by a unit of appropriate design, or by application of appropriate loading corrections to the ratio-correction-factor and phase angle. However, due to the simplicity of the circuitry and infrequent requests for calibrations at higher frequencies, a tem-

\footnotetext{
${ }^{5}$ Since maximum voltage on this divider is limited to $150 \mathrm{~V}$ at $60 \mathrm{~Hz}$, it must be connected across the test transformer rather than the reference transformer whose secondary voltage may exceed this value in some instances.
}

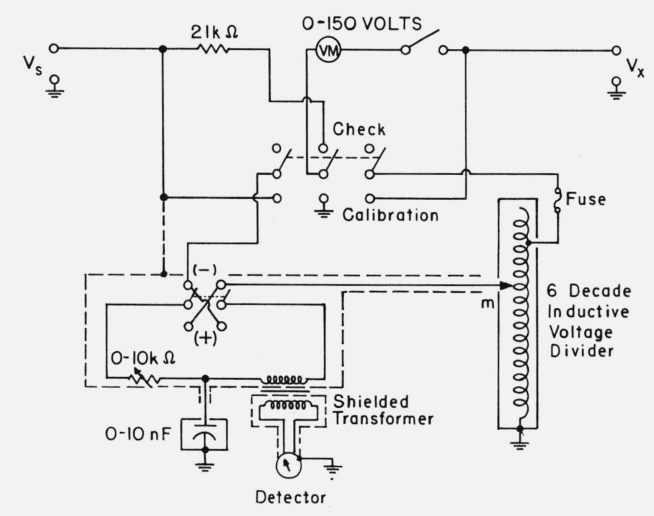

Figure 6. 


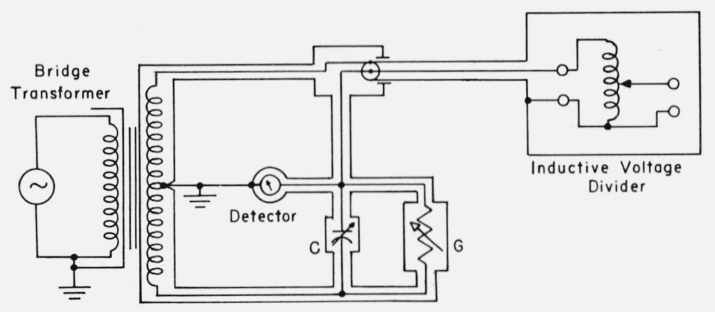

Figure 7.

porary setup is generally used at NBS. This consists of a high frequency divider, commercially available air capacitors, and an a-c decade resistor.

The input impedance of the inductive voltage divider was measured in a capacitive voltage divider setup; see figure 7 for circuit diagram [10]. The voltage-ratio corrections and phase-angle errors were obtained by a tracking method [11]. However, for a well designed inductive voltage divider, these corrections are usually $<0.2 \mathrm{ppm}$ of input in ratio linearity deviation and $<8 \mu \mathrm{rad}$ of input in phase deviation.

The detector circuit consists of a wave-analyzer used as a tuned null indicator and a specially constructed shielded transformer, shown in figure 6 . This transformer is used to match impedances and to isolate the measuring circuit from the detector circuit electrostatically. It consists of a high permeability toroidal core, a 700-turn inner primary winding, two electrostatic shields, a 3500-turn outer secondary winding, and an overall Mu-metal case. The use of a toroid minimizes the magnetic pickup from stray fields in the laboratory.

The polarity checking voltmeter is a $0-150 \mathrm{~V}, 3$-in. panel-type instrument of 3 percent accuracy. The $21 \mathrm{k} \Omega$ resistor matches its internal resistance. The voltmeter indicates the secondary voltage of the test transformer when the selector switch is in the "CALIBRATION" position.

Figures 8 and 9 show the exterior and interior of the comparator. The cylindrical shield shown in the interior view encloses the $10-\mathrm{k} \Omega$ resistor.

\subsection{Accuracy Determination}

After the completion of the comparator, the calibration of each component was rechecked.

The accuracy of the completed comparator was verified by the following procedures: (1) connecting a single voltage source to both " $V_{s}$ " and " $V_{x}$ " terminals to obtain the zero corrections for $C$ and $R$ and the unity correction for dial settings of the inductive voltage divider; (2) calibrating several pairs of transformers of the same ratio to reference transformers of appropriate ratios, thus determining the relative ratiocorrection-factors and phase-angle errors with several values of secondary burden for each pair of transform-

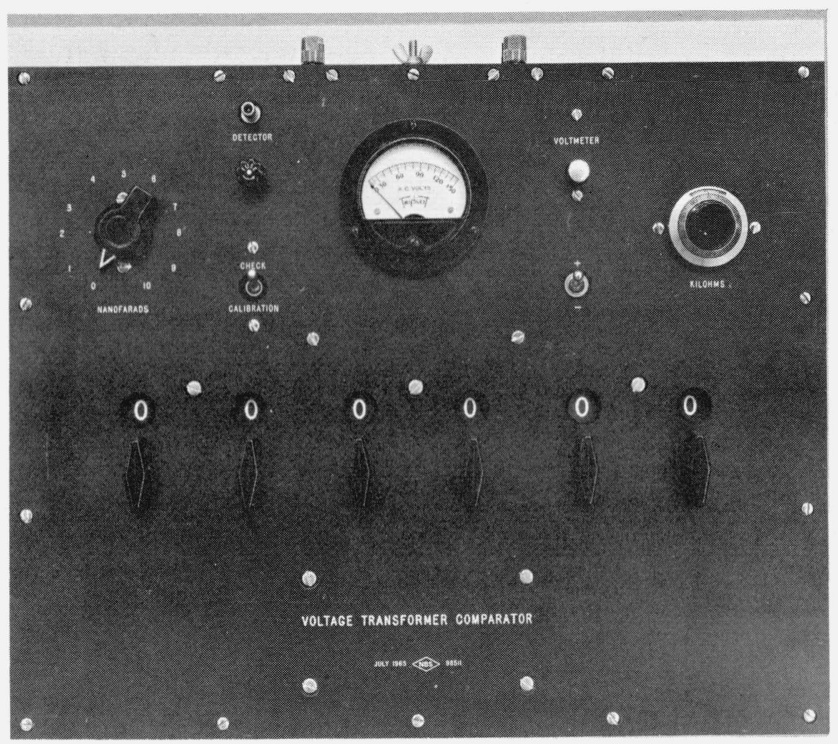

Figure 8.

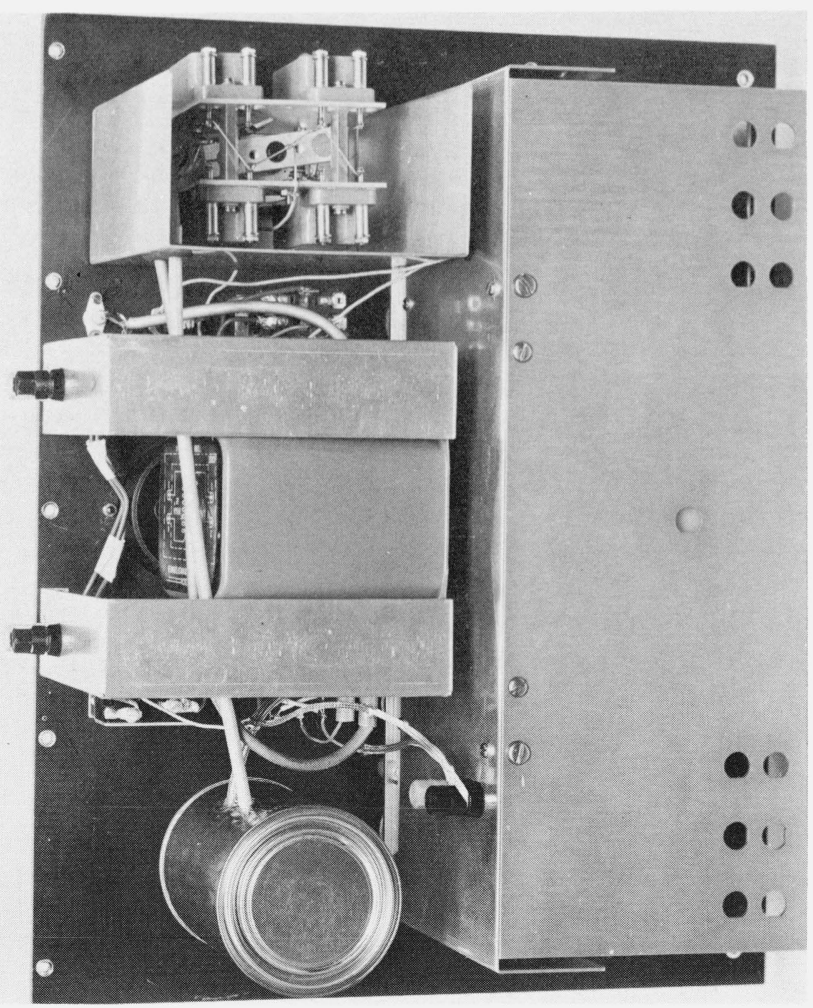

Figure 9. 
ers; (3) comparing each pair of these calibrated transformers to each other with the comparator alternately as reference and test units and with various combinations of previously used secondary burdens; and (4) connecting a single voltage source to " $V_{x}$ " terminals and to the input of a calibrated inductive voltage divider, whose output is connected to " $V$ " terminals in order to check the fractional ratio measurements.

\subsection{Transformer Testing Procedure}

A reference transformer is selected so that $N_{s} \geqslant N_{x}$. The selector switch is set to "CHECK" position; and the ratio, $m$, is set to equal $N_{x} / N_{s}$. The secondaries of the test and reference transformers are connected to " $V_{x}$ " and " $V_{s}$ " terminals respectively. With the transformers energized and the voltmeter switch depressed, the voltmeter indicates $\left(V_{s}-V_{x}\right) / 2$ if the polarities of $V_{s}$ and $V_{x}$ are correctly connected to the comparator. [The polarity of $V_{x}$ must be reversed if voltmeter indicates $\left(V_{s}+V_{x}\right) / 2$.] The test proceeds with the selector switch turned to "CALIBRATION." $V_{x}$ is now indicated on the voltmeter.

If $N_{x}>N_{s}$ by more than 10 percent, the test can still be made, but the reference transformer must be connected to " $V_{x}$ " terminal and the test transformer to " $V_{s}$ ". At the balanced condition, the ratio, $m$, will indicate the reciprocal of the relative ratio-correctionfactors, and the relative phase-angle switch will show the negative of the markings as shown in figures 6 and 8.

\subsection{Consideration of Errors}

An examination of errors requires a careful appraisal of all components of the measuring circuit as well as a justification of estimations, even though in this case one would expect most of the associated errors to be negligible.

The first to be considered are those possible errors concealed through approximation in developing the expressions for obtaining the ratio-correction-factor and phase-angle relations, where the effects of the residual of the $10-\mathrm{k} \Omega$ resistor and the dissipation factor of the decade capacitor are ignored. Approximate magnitudes of these errors can be obtained by adding the estimated equivalent residual reactance across $R$ and estimated equivalent series resistance to $C$. These effects were found to be less than $0.5 \mathrm{ppm}$ for ratio and $5 \mu \mathrm{rad}$ for phase angle at the limits of the phase angle range $( \pm 38 \mathrm{mrad})$ at $60 \mathrm{~Hz}$.

A second source of uncertainty is in the calibration of the inductive voltage dividers. However, past experience indicates that errors should not exceed $0.2 \mathrm{ppm}$ of input in ratio linearity deviation and $2 \mu \mathrm{rad}$ of input in phase deviation [12].

Errors caused by the leads, switch contacts, and imperfect shields are estimated to be $<0.5 \mathrm{ppm}$ and $<2 \mu \mathrm{rad}$.

The last important uncertainty that should be considered is in obtaining the transformer secondary load corrections. Such errors are estimated to be $0.5 \mathrm{ppm}$ and $3 \mu \mathrm{rad}$ for each transformer.

Finally, one should add 3 times the standard deviations $(0.3 \mathrm{ppm}$ of ratio and $1 \mu \mathrm{rad})$ of the results $\mathrm{ob}$ tained in repeated balances of the comparator to the combination of the systematic-error estimates.

\begin{tabular}{|c|c|c|c|c|}
\hline \multirow{2}{*}{ Source of errors } & \multicolumn{2}{|c|}{$\begin{array}{l}\text { Uncertainties to } \\
\text { ratio-correction- } \\
\text { factor }\end{array}$} & \multicolumn{2}{|c|}{$\begin{array}{c}\text { Uncertainties } \\
\text { to phase } \\
\text { angles }\end{array}$} \\
\hline & $n$ & $n^{2}$ & $n$ & $n^{2}$ \\
\hline & ppm & & $\mu \mathrm{rad}$ & \\
\hline $\begin{array}{l}\text { From assumptions made in } \\
\text { equations }\end{array}$ & 0.5 & 0.25 & 5 & 25 \\
\hline $\begin{array}{l}\text { Calibration of the inductive } \\
\text { voltage divider }\end{array}$ & .2 & .04 & 2 & 4 \\
\hline $\begin{array}{l}\text { Obtaining secondary load cor- } \\
\text { rections }(S)\end{array}$ & .5 & .25 & 3 & 9 \\
\hline $\begin{array}{l}\text { Obtaining secondary load cor- } \\
\text { rections }(X)\end{array}$ & .5 & .25 & 3 & 9 \\
\hline Leads and contacts & .5 & .25 & 2 & 4 \\
\hline $\begin{array}{l}\sum n^{2} \\
\left(\sum n^{2}\right)^{1 / 2} \\
3 \times \text { standard deviations }\end{array}$ & $\begin{array}{l}1.02 \\
0.90\end{array}$ & 1.04 & $\begin{array}{l}7.1 \\
3.0\end{array}$ & 51 \\
\hline Maximum error & 1.9 ppm & & $10.1 \mu \mathrm{rad}$ & \\
\hline
\end{tabular}

\section{Conclusion}

A high accuracy inductive comparator has been developed for measuring the relative voltage ratio and phase angle of voltage transformers and testing sets at power and audio frequencies. An overall accuracy of $<2 \mathrm{ppm}$ in ratio measurements and $<10$ $\mu \mathrm{rad}$ in phase angle at 60 and $400 \mathrm{~Hz}$ is achieved, with a slight reduction in the accuracy attainable at higher audio frequencies.

The simple circuit does not require any special equipment. Only a few ranges of reference transformers are required, since fractional ratios from $1 / 1$ to $1 / 4$ may be measured as readily as ratios near unity.

This inductive comparator could replace testing sets now in general use. It is compact and portable, has a broader useful frequency range, and exceeds the accuracy of many commercial sets presently available by one or two orders of magnitude.

The resistive comparator will continue to be used at NBS for calibration of voltage transformers at 25 $\mathrm{Hz}$ at which frequency the operating voltage is usually above the maximum operating voltage of the inductive comparator.

The author acknowledges the valuable help and advice received from Mr. F. L. Hermach, Chief, Electrical Instrument Section, NBS. Mr. R. J. Berry contributed skillfully in constructing the new comparator. 


\section{References}

[1] F. B. Silsbee and F. M. Defandorf, A transformer method for measuring high alternating voltages and its comparison with an absolute electrometer, J. Res. NBS 20,317-336 (1938) RP1079.

[2] W. K. Clothier and L. Medina, The absolute calibration of voltage transformers, Proc. Institution of Electrical Engineers, 104A, 204-214 (1957).

[3] F. K. Harris, W. C. Sze, N. L. Kusters, O. Petersons, W. J. M. Moore, An international comparison of voltage transformer calibrations to $350 \mathrm{kV}$, IEEE Trans. on Communication \& Electronics No. 70, pp. 13-19 (1964).

[4] Private communication from F. K. Harris, Chief, Absolute Electrical Measurements Section, NBS.

[5] F. K. Harris, Electrical Measurements (John Wiley and Sons, Inc., New York, N.Y.) pp. 410-411, 565-569 and 611-612 (1951).
[6] W. Duddell and T. Mather, Improvements in non-inductive resistance, British Patent No. 5171 (1901).

[7] F. B. Silsbee, A shielded resistor for voltage transformer testing, Bull. BS 20, 489-514 (1926).

[8] Private communication from F. K. Harris and B. L. Dunfee, NBS.

[9] H. B. Brooks and A. B. Lewis, Improved continuously variable self and mutual inductor, J. Res. NBS 19, 493 (1937) RP1040.

[10] M. C. McGregor, J. F. Hersh, R. D. Cutkosky, F. K. Harris and F. R. Kotter, New apparatus at NBS for absolute capacitance measurement, IRE Trans., Instr., I-7, 253-261 (1958).

[11] NBS Technical News Bulletin, 49, 1 (1965).

[12] W. C. Sze, A. F. Dunn, and T. L. Zapf, An international comparison of inductive voltage divider calibrations at 400 and 1000 hertz, IEEE Trans. on Instr. and Meas. IM-14, No. 3, 124-131 (1965).

(Paper 69C4-206) 